\title{
Integrated Information System of Monitoring and Management for Heart Centers
}

\author{
Adrian Brezulianu \\ "Gheorghe Asachi" Technical University of Iasi and \\ Greensoft Ltd. \\ Iasi, Romania
}

\author{
Monica Fira \\ Institute of Computer Science \\ Romanian Academy \\ Iasi, Romania
}

\begin{abstract}
The integrated information system presented in this paper is focused on increasing the quality of the medical services offered to the patients by rendering the activities more efficient and by corroborating the family doctors and the cardiologists' efforts. In order to accomplish the mentioned objectives we propose implementing an integrated information system by means of which the integrated management of the medical data will be done. In addition, it has been offered a collaborative instrument, patient oriented, that it will be used both by the family doctor and by the specialist doctor.
\end{abstract}

Keywords- ECG; Monitoring; Management; Integrated Information System.

\section{INTRODUCTION}

According to the World Health Organization [1], the cardiovascular diseases are considered to be the leading cause of death in the world. According to the same WHO report, the countries with a low and medium standard of life are seriously affected in comparison with the rest of the states, registering a cardiovascular disease death rate of $82 \%$. In Romania, deaths caused by the cardiovascular diseases are on the first place and Rumania is on the 4th place among the European countries, first place being taken by Russia, the second one by Bulgaria and the third one by Hungary. Taking into consideration these statistical data, a medical management is necessary in order to offer the cardiovascular diseases treatment a special place. The extension of the cardiac medical services to the rural areas, where no medical personnel specialized in cardiology can be found, represents a possible solution.

The cardiovascular diseases represent the most frequent cause of death in the economically developed countries, with a rapid ascendance, mostly in those in the developing process. It is appreciated that from 55 millions deaths registered in the world annually, approximately $30 \%$ are caused by the cardiovascular diseases. The mortality curve due to this disease registered in the last years a great difference between Central and Easter European countries - where it reached a high pick and the Northern and Western European countries - where it is dropping. [1]

To a world level, we can estimate that there are a total of 1.5 milliard adults with high blood pressure, which represents 333 millions cases in developed countries and 639 million cases in developing countries. Taking into consideration that $80 \%$ of the world population live in developing countries we can appreciate that the world prevalence of HBA will increase from $20 \%$ to $30 \%$ in the next years. The 2002 report of the World Health Organization appreciates that over $50 \%$ of the coronary diseases and approximately $75 \%$ of the strokes are due to increased systolic BP over the minimum theoretical value of $115 \mathrm{mmHg}$. [2]

Most recently, the INTERHEART study estimates that almost $22 \%$ of the acute coronary syndromes in Western Europe and 25\% in Central and Eastern Europe are due to the high blood pressure, emphasizing that the patients with HBP have a double risk of suffering an acute coronary event than the people with normal BP.

The paper initiates the presentation of an integrated information system dedicated to electrocardiographic records, by means of which the medical data management is to be improved based on a patient-oriented collaborative instrument that can be used in common by family doctors and cardiologists.

\section{INFORMATIC SYSTEM ARCHITECTURE AND FUNCTIONAL DESCRIPTION}

The proposed information system has a modular architecture with specific functions for every module. Thus, the proposed structure (presented in Figure 1) consists in the following three software modules:

1. ECG Cabinet Module

2. ECG WS Module

3. ECG Monitoring Module

\begin{tabular}{|c|c|c|c|}
\hline $\begin{array}{l}\text { ECG Cabinet Module (family } \\
\text { doctor) }\end{array}$ & $\begin{array}{c}\text { ECG } \\
\text { Cabinet }\end{array}$ & $\begin{array}{c}\text { ECG } \\
\text { Cabinet }\end{array}$ & $\begin{array}{c}\text { ECG } \\
\text { Cabinet }\end{array}$ \\
\hline ECG WS Module & \multicolumn{2}{|c|}{ ECG WS } & DB \\
\hline ECG Monitoring Module & \multicolumn{3}{|c|}{ ECG MONITORIZARE } \\
\hline
\end{tabular}

Figure 1. The Structure of Integrated Information System of Monitoring and Management

The information system should run distributed as follow:

- In the family doctor cabinet, as a distinct module (ECG cabinet) installed on a computer or laptop connected to an ECG device; ECG device will be controlled by means of this module; the results of the ECG investigation will be recorded and associated automatically with the patient. Initially data related 
with ECG investigations will be stored locally in a first phase, in a database; If an Internet connection is available, the results of the ECG investigations will be transmitted automatically to a central server for parallel processing and monitoring;

- On a central server (ECG WS and DB - Web Server and Database), where the centralization, the aggregation and the running of previous diagnosis algorithms and alerts on the base of the results of the received investigations will be carried out;
After the family doctor realizes the EKG analysis, by means of the application and the data is transmitted over Internet to the monitoring and analysis central server.
- In the permanent monitoring centre (ECG Monitoring), data regarding the patients for whom the investigation results were received together with the eventual alerts regarding the possible patient's pathology, alerts generated by the central server. The functional design of the proposed system is presented in the Figure 2.

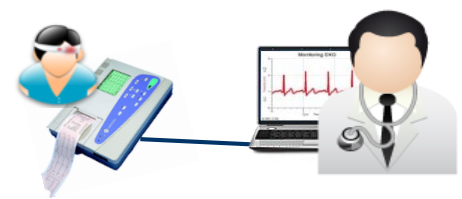

Family doctor cabinet

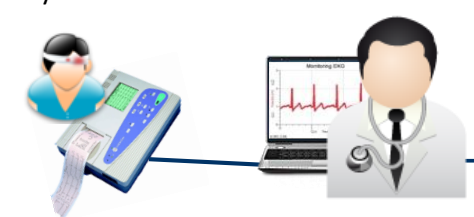

Family doctor cabinet

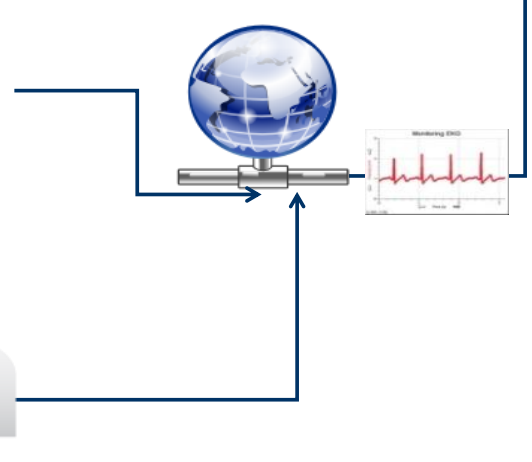

(1)

Central Monitoring Terminal

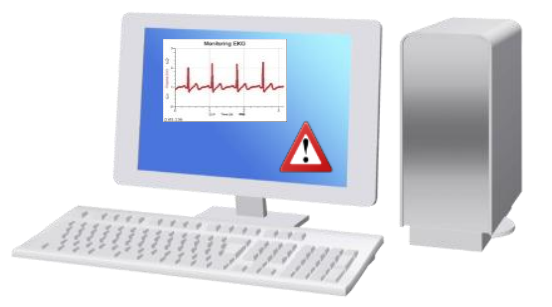

Figure 2. The functional design of the Integrated Information System

\section{A. ECG Cabinet Module / Family Doctor (FD)}

This software component is a desktop application designed to run on a computer from the family doctor's cabinet. The role of this component is to maintain records on the patient medical record, to record output results of the ECG medical equipment, in electronic format, and associate them with the patient medical record followed by the immediate transmission of this data to the monitoring centre.

\section{1) Necessary configurations}

- Installing the application on the computer in the family medicine cabinet

- Connecting the application to the medical equipment by means of the serial cable which connects the device with the computer where the application runs;

- The serial port configuration

- Installing the security certificate allocated to the family doctor

2) The main functionalities
- Maintaining the patient medical record;

- Communicating with the help of the medical equipment for ECG investigations ;

- Synchronous recording of the ECG investigation results and their automatic association with the patient medical record and his/hers medical history.

- Displaying the investigation results having the possibility of making various analyses on data by means of rescaling and measuring of the signals.

- Transmitting data that refers to the patient medical record and the investigations made in order to synchronize with a central database.

- Automatically receiving from the monitoring centre of possible specialist doctors' warnings and recommendations and this data to the patient medical record and the referred investigations. 
- $\quad$ Receiving the patient medical history from the central database. This medical history can be the result of past investigations, in other cabinet.

\section{3) Integrations}

- With a medical equipment to realize ECG investigations by means of a direct serial cable internet connection of the equipment to the computer where the software runs;

- With the central service for data synchronization ECG WS - using the internet connection and last generation communication technologies based on web services.

\section{4) Using technologies}

In order to develop this software component last generation, software technologies that assure its compatibility with the last generation operating and calculation systems were used, as well as, the necessary minimal knowledge for realizing this configuration for using it.

For communicating on the Internet web service technologies are used in order to synchronize with the central data base, using standard communication protocols that proved their efficiency by their implementation with other public systems.

\section{5) Data security}

In order to assure the confidentiality of the personal data and the investigation results, during the transmission of these data to the central database, crypting and data integrity assurance technologies are used.

For the system access, for every doctor a digital certificate will be generated by means of which he/she will be identified in the system and will be able to encrypt the transmitted data.

In addition, a medical history will be maintained with the activity of each user in the system on the base of which statistical analyses can be made for identifying some possible intents to compromise the data.

\section{B. ECG WS Module}

This component of the information system will be installed on a server that will represent the data confluence place of all the family doctor cabinets, data that will be stored on this server in a database. Immediately after receiving, the data will be offered to the specialist doctors by means of ECG Monitoring Module. Besides the expected data (patient medical record + the realized investigation in electronic format), the system will generate some alerts as a consequence of a preprocessing process and possible pathologies identification.

\section{1) The main functionalities}

- The management of the access right inside the system.

- Certifying and authorizing the system users

- Communication with ECG Cabinet applications installed in the family doctor cabinets for receiving the patient medical records and the realized ECG investigation results.

- $\quad$ Storing data in the database.
- Realizing pre-processing operations to identify the possible pathologies and alerts sent to the specialist doctor.

- Synchronous Data Transmission to the ECG Monitoring Component operated by the specialist doctor.

- $\quad$ Specialist doctor generated messages transmission to the ECG Cabinet referring to an investigated patient.

- Maintaining access and activity logs inside the system.

\section{2) Integrations}

- With the ECG Cabinet module, by means of an Internet connexion for undertaking the patient medical record and the ECG investigation results;

- With the ECG Cabinet module, for transmitting to the specialist doctor the investigations received from the family doctor;

- With the ECG Cabinet module, for transmitting the reply of the specialist doctor to the family doctor.

3) Using technologies

JAVA, J2EE, AXIS WS, SQL

\section{ECG Monitoring Module}

This software component is implemented under the form of a desktop application that runs on one or several computers inside the monitoring centre [3- 8]. By means of this application, the specialist doctor has access to the medical record data, the current ECG investigation as well as to the history of all ECG investigations made for the patient in the past, no matter the place where these were realized (another family doctor). The specialist doctor by means of these software instruments has direct access to software instruments to visualize and analyze the investigation result based on which he/she can make recommendation or warnings that can be instantly transmitted to the family doctor cabinet where the specific investigation was made.

\section{1) Extensibility. Regionalization}

According to the number of family medicine cabinets registered in the system and the intensity of their activity, with the object of maintaining the efficiency of the answering time, it is possible the allocation of specialist doctors depending on groups of cabinets, the allocation criteria being dynamic (region, locality, area, etc.). Thus, each specialist doctor will receive on his/hers screen only the messages of the cabinets assigned to that.

\section{2) Automat signal processing and alert engendering}

When receiving the results of an ECG investigation on the central server these data pass by some pre-processing operations by means of which the following activities are carried out:

- The detection of the $\mathrm{R}$ wave with the object of subsequent annotation of the diagram seen by the specialist doctor. [9] 
- The cardiac frequency calculation with the emission of warnings on a frequency higher than 130 beats per minute or lower than 40 beats per minute.

- The variability cardiac rhythm calculation on a number of 6-8 cardiac complexes. The engendering of warnings at variability $20 \%$ higher or lower, together with graphically marking the specified area.

- QRS complex detection [10]

- Medium term calculation of the QRS complex. Warning engendering at a average value of the QRS complex term bigger than $120 \mathrm{~ms}$.

- The detection of the $\mathrm{S}, \mathrm{T}$ and $\mathrm{P}$ waves with the beginning and the end of these waves.

- Warning at a medium value of the ST segment $2 \mathrm{~mm}$ bigger than the izoelectric line or $2 \mathrm{~mm}$ smaller than the izoelectric line.

- Heart beat classification and graphical display according to following classes: $0=$ Atrial premature beat, $1=$ Normal beat, $2=$ Left bundle branch block beat, 3 = Right bundle branch block beat, $4=$ Premature ventricular contraction, $6=$ Fusion of ventricular and normal beat, $8=$ Paced beat, $9=$ Fusion of paced and normal beat

\section{3) The main functionalities}

- The download from ECG WS of data regarding the patient's medical record and the investigation results;

- Displaying the data resulting from the pre-processing made on the central server based on priorities resulted after establishing an emergency degree;

- The specialist doctor offers instruments to analyze and process the ECG investigation result (amplitude scaling, time, QRS detection, measuring).

- The registration of observations, recommendations and warnings of the specialist doctor and their registration by means of ECG WS to the family medicine cabinet where they would be automatically displayed in the ECG Cabinet.

\section{4) Integrations}

With ECG WS for taking over the data related with the patient's medical record, the investigations result and for transmitting the observations, recommendations and warnings related to current investigation to the family doctor.

\section{Data transfer design}

The general patient-oriented intercommunication diagram is presented in the figure 7, where the family doctor takes from the patient the necessary data for filling in the medical record, together with the ECG registration, and transmits them to the cardiologist along with a message containing questions and comments related to the patient' situation. The specialist doctors evaluate the received data and answers the questions and/or prescribe a treatment scheme or specifies the necessary additional investigations.

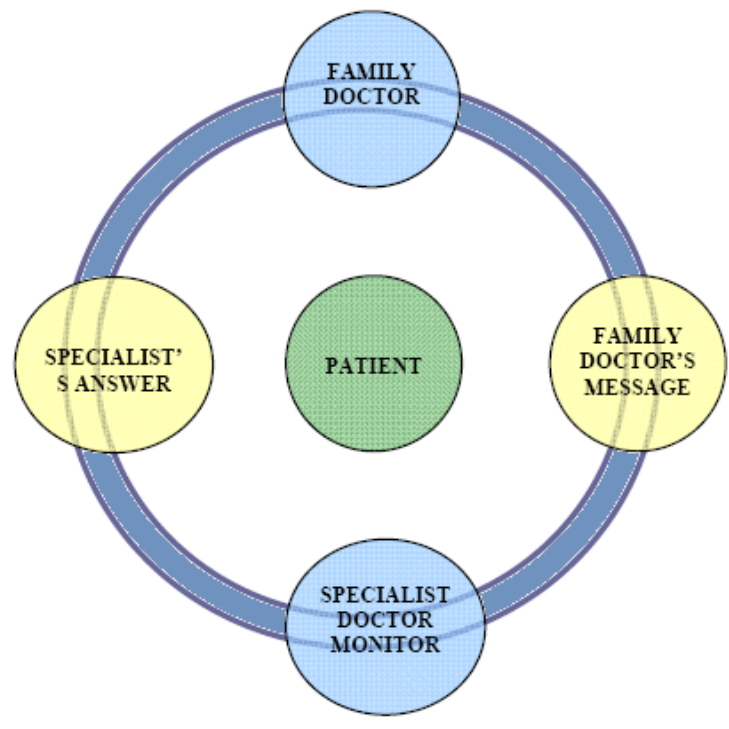

Figure 3. Patient-oriented communication
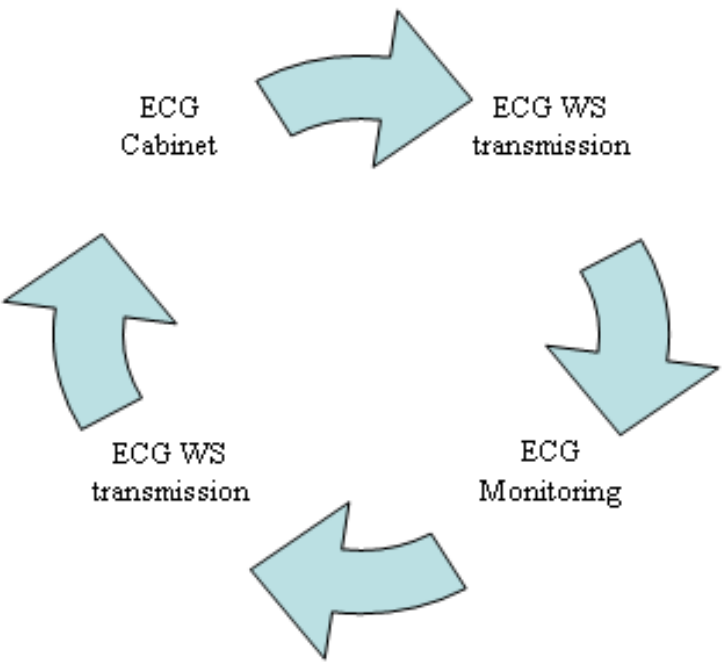

Figure 4. Communication between the information system components

The main intercommunication characteristics (data transfer) are the following:

- Data transfer to the CENTRAL server is done respecting the in force standards regarding the data security assurance; For this encryption algorithms based on public/private key are used;

- Any data transmission between the family doctor cabinet and the central server is made after a previous authentication of the transmitter's identity;

- The data transfer is made taking into consideration the restrictions regarding the quality of the available internet connection from the family medicine cabinets;

- The data transfer from the family medicine cabinets to the CENTRAL server is made using data blocks of 
configurable sizes according to the known quality of the speed and available internet connection stability;

- This solution is absolutely necessary for implementing the transmission return functionality in case of scenarios like:

- Low Speedy Internet connection;

- Instable Internet Connection;

- Transmitted data sample of relatively big size $(2-3 \mathrm{MB})$;
- The minimum size of the data block is established to $10 \mathrm{~KB}$;

- Data retransmission will be resumed till the confirmation of receiving it on the CENTRAL server;

- After the complete reception of data on the CENTRAL server it will be processed in order to transfer the registrations into the database;

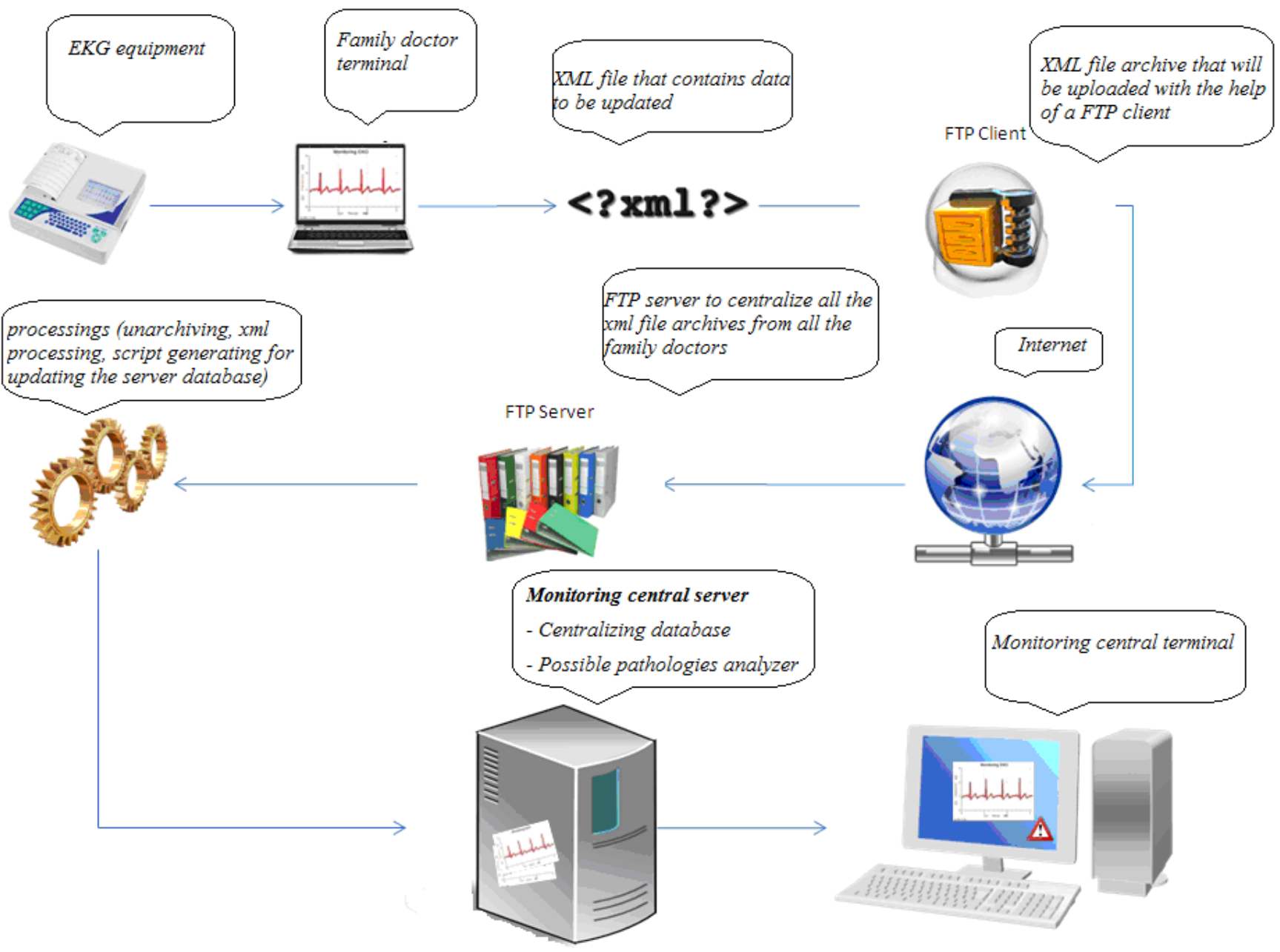

Figure 5. General intercommunication scheme

1. The ECG equipment is controlled by means of the ECG cabinet with the purpose of achieving the ECG investigation and collecting within the database the investigation results.

2. When the application is synchronized, an xml local file is created which contains the necessary data for updating the information from the server (patient information, investigation information, doctor information). A record regarding the date/hour of synchronization is saved in the database, thus being able to reproduce a history of synchronizations. Observations regarding the file structure:
- In order to optimize the data transfer, the $\mathrm{xml}$ will; have to have a dimension as reduced as possible.

- The file contains only patients who have modified data, who have registered new investigations or who were not synchronized at all on the server (new patient).

- The file contains only the investigations which are not synchronized on the server (new record) 
- Within the <graphic> are saved all the points that outline the ECG graphic, separated by the "\#” separator.

3. The xml saved file is archived with the help of an archiver (Win Zip, Win Rar). The result is a file of archive type which has a much smaller dimension than the previous one (We can obtain an archive with a 7 times smaller dimension)

4. With the help of a FTP client (File Transfer Protocol) the transfer occurs from the computer/laptop which has installed the ECG cabinet (MF) module, with the help of the internet connection, on the FTP server which is located on the machine that has the COLLECTION/ANALYSIS Module installed. Through this type of server, the archive reaches the server safely. In the case that the internet connection is unstable, the synchronization is resumed from where it had stopped when the internet connection is reestablished. The data is encrypted by means of SSL protocole. For this encrypting 2 keys are necessary: a public one that everybody knows and a private one known only by the server.

5. After the synchronization, the data reaches the server. Here are centralized all the archives coming from all the ECG cabinet (MF) terminals. The files on this folder have unique names composed of the national identification number of the Family Doctor whose data concerning the patients are synchronized as a suffix and the date and hour when the synchronization occurs. The files that are transferred in totality and processed receive the " $\mathrm{T}$ " prefix which indicates that the data from this archive was synchronized and are kept as back-up.

Example

$$
\begin{aligned}
& \text { 1650921336402_27_09_2010_17_58_22.zip } \\
& \text { 1700201256213_01_06_2010_12_01_33_T.zip }
\end{aligned}
$$

6. When an archive is transferred in totality on the server, the file processing begins. The $\mathrm{xml}$ file is unachieved. The latter is placed and with the information it contains, the insert and update scripts are created. The scripts are run in the server database and have the role to save the new data come from the ECG cabinet terminal (MF).

7. The data automatic analysis algorithms are created. They detect the eventual anomalies within the investigations corresponding to the newly synchronized patients and save in the database new records corresponding to the pre-diagnostic.

8. On the live monitoring interface, the new patients synchronized in the order of pathologies priority will appear. Visual and sound alerts are transmitted to attract the attention on the cases that require immediate attention.

\section{CONCLUSIONS AND FUTURE WORK}

The information system presented in this paper and which has as purpose improving the management of medical information by increasing the quality of the medical services offered to the patients supposes a series of activities and namely:

- Generating information instruments of doctor's assistance in the interpretation of the electrocardiograph investigations results

- Generating and maintaining a centralized patient history from the point of view of the disorders and investigations carried out;

- Implementing a collaborative instrument usable by the family doctors and the specialist cardiologist physicians through which the medical information is efficiently disseminated in the patient's interest;

- Implementing certain signal processing dedicated to the ECG signals with the purpose of identifying the possible pathologies and issuing warnings to the specialist doctor and the family doctor

- Generating a centralized medical database.

In future, we intend working with as many medical centers to implement the presented system and customize the application according to specific requirements of each center.

\section{ACKNOWLEDGMENT}

This work has been supported by the Greensoft Ltd. company, Romania.

\section{REFERENCES}

[1] WHO (World Health Organization) - www.who.int/en/

[2] AHA (American Heart Association)., (2008). Heart Disease and Stroke Statistics - 2008

[3] Ince, T. , Kiranyaz, S., Gabbouj, M. , A Generic and Robust System for Automated Patient-Specific Classification of ECG Signals, IEEE Transactions on Biomedical Engineering, Vol. 56, Issue 5, 2009

[4] Gerencser, L. , Kozmann, G. ; Vago, Z. ; Haraszti, K. , The use of the SPSA method in ECG analysis, IEEE Transactions on Biomedical Engineering, Vol. 49, Issue 10, 2002

[5] Osowski, S. , Tran Hoai Linh, ECG beat recognition using fuzzy hybrid neural network , IEEE Transactions on Biomedical Engineering, Vol. 48, Issue 11,2001

[6] Mar, T., Zaunseder, S., Martínez, J.P., Llamedo, M., Poll, R., Optimization of ECG Classification by Means of Feature Selection, IEEE Transactions on Biomedical Engineering, Vol. 58, Issue 8, 2011

[7] Philip de Chazal, O'Dwyer, M., Reilly, R.B. , Automatic classification of heartbeats using ECG morphology and heartbeat interval features, IEEE Transactions on Biomedical Engineering, Vol. 51, Issue 7, 2004

[8] de Chazal, P., Reilly, R.B., A Patient-Adapting Heartbeat Classifier Using ECG Morphology and Heartbeat Interval Features, IEEE Transactions on Biomedical Engineering, Vol. 53, Issue 12, 2006

[9] Negoita, M., Goras, L., (2004). The R-wave Detection with Low Computation Complexity Based on the Pan-Tompkins Algorithm. Buletinul Institutului Politehnic din Iasi, Tomul L (LIV), Fasc. 3-4, Electrotehnica, energetică, Electronică

[10] Negoita, M., Goras, L., (2004). A Robust Algorithm for Accurate QRS Complex Detection. Buletinul Institutului Politehnic din Iasi, Tomul L (LIV), Fasc. 1-2, , Electrotehnica, energetică, Electronică 
